\title{
A LINEAR PARAMETER-VARYING MULTIOBJECTIVE CONTROL LAW DESIGN BASED ON YOULA PARAMETRIZATION FOR A FLEXIBLE BLENDED WING BODY AIRCRAFT
}

\section{F. Demourant and G. Ferreres}

ONERA

2 Avenue Edouard Belin BP74025

Toulouse 31055, France

\begin{abstract}
This article presents a methodology for a linear parameter-varying (LPV) multiobjective flight control law design for a blended wing body (BWB) aircraft and results. So, the method is a direct design of a parametrized control law (with respect to some measured flight parameters) through a multimodel convex design to optimize a set of specifications on the full-flight domain and different mass cases. The methodology is based on the Youla parameterization which is very useful since closed loop specifications are affine with respect to Youla parameter. The LPV multiobjective design method is detailed and applied to the BWB flexible aircraft example.
\end{abstract}

\section{INTRODUCTION}

Number specifications are involved to control a flexible aircraft. These specifications can be very different: handling qualities, load alleviation in the frequency and/or time domain representation, command effort with saturation and rate limiters, comfort, and robustness [1-3]. To meet these different kinds of specifications, the Youla parameter design, namely, the convex synthesis [4] is involved. This approach is very interesting for several reasons. All stabilizing controllers can be parametrized thanks the Youla parameter and the closed loop transfer functions are affine with respect to Youla parameter. Then, all specifications which correspond to the constraints on closed loop transfer functions can be rewritten as convex optimization problem. Finally, the problem solved is convex which guarantees the globality of the optimum found and a 
good tractability of the optimization algorithm. This last point is the most important as a specificity of a flexible aircraft is the high order of models. In brief, the convex synthesis is, clearly, a multiobjective/multicriterion control law design approach.

The second important point is to keep the performance level for full flight domain and different mass/fuel cases. This point leads to schedule a control law with measurable parameters which impact the behavior of the aircraft. A useful representation to make appear naturally the Youla parameter is the estimated state feedback structure. By this representation, a natural LPV controller is obtained since a parametrized model is embedded in the observer. A typical parametrization is a linear fractional transformation (LFT) representation of the model to control where the $\Delta$ block contains scheduling parameters. Let point out two important points. Firstly, it is not necessary to schedule the observer and state gains and/or the Youla parameter if the closed loop behavior is satisfactory. Secondly, the LFT representation, which can be difficult to determine with high order models and/or numerous scheduling/robustness parameters [5], is a possible representation, but other parametrizations such as a polynomial parametrization can be used for the observer.

The study of the BWB aircraft control is a part of the European project ACFA2020. The objective is to show that it is possible to improve significantly the performance of a BWB aircraft with respect to a classical one. For the control of a flexible aircraft as here a BWB aircraft, there are two sets of specifications. The first set of specifications concerns the handling qualities, i. e., the behavior of the aircraft with pilot and flight control law. Specifications will be widely described in the paper. It is important to note that it is not expected that all handling qualities specifications are satisfied by the feedback. If the feedback is considered as satisfactory, it is possible and necessary to use a feedforward to shape time domain response in order to meet fully handling qualities specifications. The second set of specifications concerns the load alleviation on critical load outputs. Typically, the main objective is to decrease the load level for the wing root bending moment (WRMX) under the constraint to satisfy actuators saturations and rate limiters and not to increase the wing root vertical force (WRFz). The last specification concerns the improvement of the passangers comfort. This specification consists in minimizing the $\mathrm{H}_{2}$ norm of cabin accelerations.

For the rigid part, a linear-quadratic-Gaussian (LQG) methodology is involved. This methodology is very interesting in the present context because it makes appear the structure of the estimated state feedback naturally. Of course, from theoretical point of view, any dynamic feedback output can be put under an estimated state feedback form [6]. But this additional step is not obvious to involve and can lead, in the context of an LPV context, to controllers which are not interpolable with a suitable behavior. The results obtained in terms of closed poles placement and time domain simulations are satisfactory without schedul- 
ing observer and state gains. But, of course, this controller is an LPV controller due to the fact that the observer is parametrized. This controller represents the initial stabilizing LPV controller. Now, the Youla parameter is designed to meet specification on the flexible part. Finally, the load alleviation, which is the main objective, is obtained while satisfying contraints on WRFz and actuators with a comfort improvement. At last, when the feedback is synthesized, a feedforward is designed to satisfy completely the handling qualities specifications.

\section{STRUCTURE OF THE CLOSED LOOP}

The longitudinal model of a BWB aircraft is of order 23. This model includes 4 rigid states (pitch oscillation and phygoid modes), 6 flexible modes, i. e., 12 flexible states and 7 lag states. This model is composed of two parts:

(1) a rigid part which corresponds to the handling qualities model; and

(2) a flexible part which corresponds to the aeroelastic model.

The structure of the closed loop for the longitudinal control of a civil aircraft is the following one. The measures used by the controller are $\mathrm{Nz}_{\text {law }}, q$, and $\mathrm{Nz}_{\mathrm{CG}}$ where $q$ and $\mathrm{Nz}_{\mathrm{CG}}$ represent, respectively, the pitch rate and the vertical acceleration on the center of gravity. These two outputs are used to obtain satisfactory results for the handling qualities. The measure $\mathrm{Nz}_{\text {law }}$ is defined as follows:

$$
\mathrm{Nz}_{\text {law }}=\frac{\mathrm{Nz}_{\mathrm{lw}}+\mathrm{Nz}_{\mathrm{rw}}}{2}-\mathrm{Nz}_{\mathrm{CG}}
$$

where $\mathrm{Nz}_{\mathrm{lw}_{\mathrm{w}}}$ and $\mathrm{Nz}_{\mathrm{rw}}$ represent, respectively, the vertical acceleration on the left and right wing that allows to catch the symmetric flexible modes of the wing in order to control them and then to decrease the load level and to improve the comfort for passengers. The inputs of the aircraft used by the controller correspond to the elevators (inner and outer) and the outer ailerons. The elevators allow to obtain good handling qualities and the ailerons allow to control the symmetric flexible modes. As just the longitudinal dynamic is investigated, ailerons and elevators are deflected in a symmetric way. The last input, $N z_{\text {com }}$, corresponds to the reference input.

A second-order actuator is used for each input. Besides, a second-order Padé model of $160 \mathrm{~ms}$ with an additional low-pass second-order filter are added on $q$ and $\mathrm{Nz}_{\mathrm{CG}}$. A second-order Padé model of $60 \mathrm{~ms}$ is added on $\mathrm{Nz}_{\text {law }}$. These actuators have specific characteristics since the dynamics of these actuators are very slow as indicated by Table 1 . This kind of dynamics leads to have a strong amplitude of controller output signals. Besides, as rate limiters and saturations 
Table 1 Actuators characteristics

\begin{tabular}{lcccc}
\hline Location & Dampings & $\begin{array}{c}\text { Frequency, } \\
\mathrm{rad} / \mathrm{s}\end{array}$ & $\begin{array}{c}\text { Position } \\
\text { limits }\end{array}$ & $\begin{array}{c}\text { Velocity limits, } \\
\mathrm{deg} / \mathrm{s}\end{array}$ \\
\hline Elevators & 0.707 & 2.71 & {$\left[-30^{\circ}+15^{\circ}\right]$} & {$[-30+30]$} \\
Outer ailerons & 0.707 & 7.77 & {$\left[-25^{\circ}+25^{\circ}\right]$} & {$[-40+40]$} \\
\hline
\end{tabular}

Table 2 Flight cases at different true air velocities $V_{\text {TAS }}$

\begin{tabular}{cccc}
\hline Mach & $V_{1}, \mathrm{~m} / \mathrm{s}$ & $V_{2}, \mathrm{~m} / \mathrm{s}$ & $V_{3}, \mathrm{~m} / \mathrm{s}$ \\
\hline 1 & 243.6 & 248.6 & 252.9 \\
2 & 251.6 & 256.1 & 260.3 \\
3 & 259.8 & 263.7 & 267.7 \\
\hline
\end{tabular}

Table 3 Flight cases at different altitudes

\begin{tabular}{cccc}
\hline Mach & $H_{1}, \mathrm{~m}$ & $H_{2}, \mathrm{~m}$ & $H_{3}, \mathrm{~m}$ \\
\hline 1 & 10,871 & 9,031 & 7,793 \\
2 & 11,335 & 9,513 & 8,287 \\
3 & 11,777 & 9,973 & 8,761 \\
\hline
\end{tabular}

are situated before actuators, i. e., on the controller outputs, rate limiters represent strong contraints for the command effort. Data about saturations and rate limiters are given in Table 1.

Globally, the system to control is of order 37 (23 aircraft +4 actuators +10 sensors). Of course, it is necessary to add others inputs and outputs which are not used by the controller but essential to satisfy specifications such as the wind and derivative wind inputs, WRMX and WRFz outputs, and cabin accelerations to improve comfort.

The flight domain is defined by 3 Mach numbers and 3 dynamic pressures. Tables 2 and 3 provide different flight cases in true air speed and altitudes. Eight fuel cases have been considered from the case $20 \%$ to the case full fuel tank by step of $10 \%$. Finally, 9 flight cases and 9 fuel/mass cases are obtained which correspond to 81 models.

To evaluate the load level, two kinds of signals for perturbations can be considered. The first one is the turbulence which is usually represented by a linearized von Karman filter. In the considered application, this perturbation does not represent the critical perturbation in the sense that it does not lead to high load level. The second one is the discrete gust which is modeled by the following relation:

$$
U=\frac{U_{d s}}{2}\left[1-\cos \left(\frac{\pi V_{\mathrm{TAS}}}{H}\right)\right]
$$

where $V_{\text {TAS }}$ is the true air speed of the aircraft; $U_{d s}$ is the amplitude which varies from 11.9 to $19 \mathrm{~m} / \mathrm{s}$; and $H$ is the scale which lies between 9 and $152.4 \mathrm{~m} / \mathrm{s}$. This kind of perturbation leads to sizing the load levels. 


\section{PRINCIPLE OF THE CONVEX SYNTHESIS}

In this paper, the Youla, or $Q$-parametrization, is involved. The convex synthesis $[4,7]$, a Youla parameter based technique, is similar to the $H_{\infty}$ synthesis, since it allows to weight closed loop transfer matrices. Besides, it is possible to take into account a priori and explicitly time and frequency domain constraints. The advantage of this approach for the considered problem is the possibility to take into account different kinds of specifications $\left(H_{\infty}, l_{\infty}, H_{2}\right.$, etc.) very easily.

\subsection{Affinity of Closed Loop Tranfer Functions}

Let consider the classical standard form where $y(t)$ and $u(t)$ are the inputs/ outputs of the control law and $w(t)$ and $z(t)$ are the closed loop inputs/outputs to control. Typically, $w(t)$ are the reference inputs, measure noise, and nonmeasured perturbations. Outputs $z(t)$ represent any closed loop weighted signals which must be controlled by the control law; $P(s)$ represents the synthesis model with weighting functions; and $K_{0}$ represents the available control law. Two hypothesizes are necessary to use of convex synthesis methodology:

(1) the transfer matrix $P(s)$ should be proper; and

(2) the initial controller $K_{0}$ should ensure closed loop stability.

Let split transfer matrix $P$ in the following way:

$$
P=\left[\begin{array}{ll}
P_{11} & P_{12} \\
P_{21} & P_{22}
\end{array}\right] \text {. }
$$

It is possible to write the transfer matrix between $w$ and $z$ as a function of $P$ and any controller $K$ by the lower linear fractional transformation $F_{l}(P, K)$ :

$$
T_{w \rightarrow z}=F_{l}(P, K)=P_{11}+P_{12} K\left(I-P_{22} K\right)^{-1} P_{21} .
$$

In the considered synthesis problem, it is necessary to write the set of time and frequency domain specifications under mathematical criteria. For instance, frequency domain specifications can be written as the minimization of $\gamma_{i, j}$ under the frequency domain constraint:

$$
\left\|T_{w_{i} \rightarrow z_{j}}(j \omega)\right\|_{\infty} \leq \gamma_{i, j} \Leftrightarrow\left\|F_{l}(P, K)_{i, j}\right\|_{\infty} \leq \gamma_{i, j} .
$$

The problem is to determine the control law $K$ which satisfies specifications (2), which is deeply nonlinear in $K$. It will be shown that $Q$-parameterization allows to express the closed loop constraints as a linear expression in $Q$ :

$$
F_{l}(P, K)=T_{1}-T_{2} Q T_{3}
$$


where $Q$ becomes the synthesis parameter and $T_{1}, T_{2}$, and $T_{3}$ contain the poles of the initial closed loop system. In fact, $Q$-parameterization allows to substitute $Q$ to $K$ to make the optimization problem convex. The $Q$-parameterization allows to describe all $K(s)$ which stabilize the closed loop: if a control law satisfying the specifications exists then it is possible to find it by optimizing the $Q$ parameter.

It has been shown that closed loop transfer matrix is affine in $Q$ for LFT; $Q$ can be parameterized as follows:

$$
Q=\sum_{i=1}^{n} \theta_{i} Q_{i}
$$

where $Q_{i}$ are the filters whose poles are determined a priori and $\theta_{i}$ are the optimization parameters. The set of these filters is a base which is used to build $Q$. Then, the LFT can be written of the following way:

$$
F_{l}(P, K)=T_{1}-\sum_{i=1}^{n} \theta_{i} T_{2} Q_{i} T_{3} .
$$

Let assume $F_{l_{0}}=T_{1}$ and $F_{l_{i}}=-T_{2} Q_{i} T_{3}$. One obtains:

$$
F_{l}(P, K)=F_{l_{0}}+\sum_{i=1}^{n} F_{l_{i}} \theta_{i}=F_{l_{0}}+\mathbf{F}^{t} \Theta
$$

where the closed loop transfer matrix is affine in $\Theta$, vector of the decomposition of $Q$ over the base. It can be shown that frequency and time domain responses are also affine in $\Theta$. The problem can then be efficiently solved with the cutting planes method.

\subsection{Choice of a Base}

To choose a base for $Q$ comes down to determine poles, it is important to note that poles of filters are the poles of the final closed loop by property of $Q$-parameterization. In the field of system identification, numerous studies exist about the generation of these bases.

Theoretically, an infinite number of base elements is needed, but as the control law order depends on the base order, a base which order is compatible with specifications is chosen. An orthonormal base is used, called Takenaka and Malmquist base, which combines the properties of Laguerre and Kautz bases. The decomposition of $Q_{i}(s)$ is given by the following equation:

$$
Q_{i}(s)=\frac{\sqrt{2 \Re\left(a_{i}\right)}}{s+a_{i}} \prod_{k=1}^{i-1} \frac{s-\overline{a_{k}}}{s+a_{k}} ; \quad Q_{0}(s)=1, \quad a_{k} \in C^{+}
$$


where $a_{k}$ are the filters poles and are determined a priori to cover the frequency domain of the bandwidth; and

$$
Q=\sum_{i=1}^{N} \theta_{i} Q_{i}
$$

\subsection{A Structure for Youla Parameter}

One method to get a Youla parametrization is to design an initial stabilizing observer-based state feedback which is a posteriori augmented with the inputs $e$ and outputs $v$ of $Q(s)$ :

$$
\begin{aligned}
\dot{\hat{x}} & =A \hat{x}+B u+L(y-C \hat{x}-D u) \\
u & =-K \hat{x}+v \\
e & =y-C \hat{x}-D u
\end{aligned}
$$

where $K$ and $L$ represent, respectively, the state feedback and the observer gain. Finally, the control law order $K(Q)$ is the sum of the order of the initial control law $K_{0}$ and the order of $Q$.

\subsection{Initial Stabilizing Controller}

The initial stabilizing controller has been designed by a very classical LQG approach. Let remind that this approach is based on the minimization of the following criterion:

$$
\int_{-\infty}^{+\infty}\left(x^{\mathrm{T}} Q x+u^{\mathrm{T}} R u\right) d t
$$

where $x$ is the state vector and $u$ is the input signal of the system to control such as

$$
\dot{x}=A x+B u ; \quad y=C x+D u .
$$

Matrices $Q$ and $R$ are the design parameters and are chosen to satisfy the specifications. Finally, a state feedback $K$ such as $u=-K x$ is obtained. A similar formulation exists to synthesize the observer gain $L$.

\section{RESULTS}

As indicated previously, convex synthesis is done in two steps. The first one is to obtain an initial stabilizing LPV controller. From methodological point of view, 
this initial controller is designed to satisfy specifications on the rigid part. The rigid part is a forth order model with two dynamics:

(1) the pitch oscillation; and

(2) the phygoid modes.

\subsection{Handling Qualities}

Specifications concern the pitch oscillation since the phygoid is treated thanks an autothrotle which is not the objective here. But a hard constraint must be respected since the pitch oscillation control do not make the phygoid too unstable, i. e., the phygoid must remain real and the possible instability inferior to $+0.1 \mathrm{rad} / \mathrm{s}$. In other words, the phygoid can be unstable but real and very slow to be controllable by the pilot.

Specifications are the following ones:

(1) a static error null between the $\mathrm{Nz}$ command $\mathrm{Nz}_{\text {com }}$ and $\mathrm{Nz}_{\mathrm{CG}}$ for a step input;

(2) perturbation rejection must be ensured;

(3) a correct closed loop poles placement, i. e., the control law is able to reject a nonmeasured perturbation in 5 or $6 \mathrm{~s}$; and

(4) a first-order behavior for $\mathrm{Nz}_{\mathrm{CG}}$ with a step reference input on $\mathrm{Nz}_{\mathrm{com}}$. A rising time of 3 to $6 \mathrm{~s}$ is expected with a very limited overshoot on $\mathrm{Nz}$ and an overshoot maximum of $30 \%$ on $q$.

The first three specifications can be and must be satisfied only by the feedback. In fact, it is necessary to have an integrator in the controller to ensure the perturbation rejection and the null static error. Besides, the closed loop poles placement cannot be modified by a feedforward; that is why, it is necessary to satisfy with the feedback the specification concerning the perturbation rejection in 5 or $6 \mathrm{~s}$. The last specification is treated thanks a feedforward. But to make easier the design of the feedforward, it is interesting to have, with only the feedback, time domain response as closed as possible to this specification.

The structure of the two-degree-of-freedom (2DOF) controller is given in Fig. 1. Let notice the integrator pole in the controller to ensure a perturbation rejection, the feedforward which acts on only the elevators to satisfy the handling qualities specifications and the Youla parameter which uses the estimation error. 


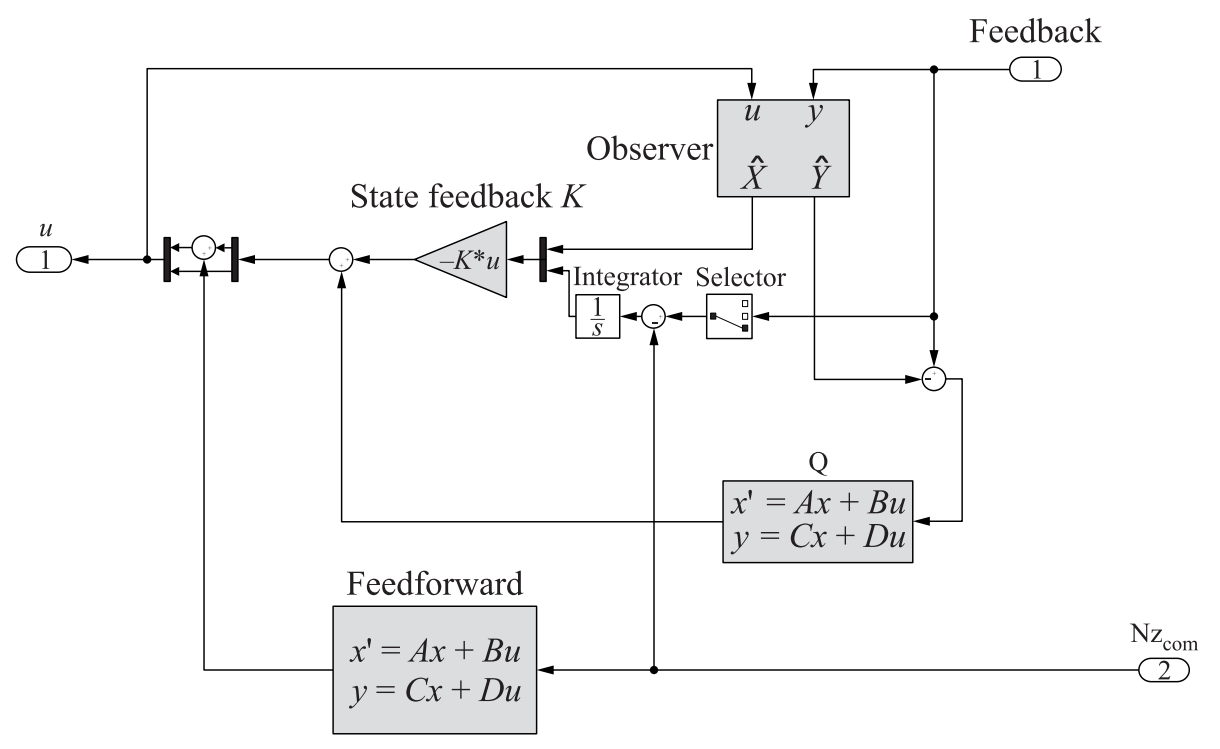

Figure 1 Structure of the 2DOF controller

\subsubsection{Design of the state-feedback controller}

The design model corresponds to the most unstable model with pitch oscillation and phygoid modes. The phygoid mode is unstable $(-0.133$ and +0.206$)$ while the damping ratio of the short period mode, namely, 0.527 , is close to the minimum value over the operating range.

The design model for the state feedback controller is the 21-state integral model (with a second-order rigid part only corresponding to the pitch oscillation) + actuator and sensor models + an integrator on the $\mathrm{Nz}_{\mathrm{CG}}$ output. Only the elevators are used.

A linear-quadratic (LQ) method is used as written previously to design the initial stabilizing controller. For the weighting matrix, $R=1$ on $u_{1}$, and for the states, the weighting matrix $Q$ corresponds to $Q=\mu_{1} c_{1} c_{1}^{\mathrm{T}}+\mu_{2} c_{2} c_{2}^{\mathrm{T}}$. The output $y_{1}=c_{1}^{\mathrm{T}} x$ corresponds to the integrator on the $\mathrm{Nz}_{\mathrm{CG}}$ output, while $y_{2}=c_{2}^{\mathrm{T}} x$ corresponds to the $\mathrm{Nz}_{\mathrm{CG}}$ output itself. For the application, $\mu_{1}=\mu_{2}=0.01$.

Finally, the results in terms of closed loop poles placement are the following ones for all models over the operating range with only the pitch oscillation:

- the integrator pole remains real in closed loop;

- the open loop real lag pole remains real in closed loop; and 
- the pitch oscillation mode, with a damping ratio of about 0.5 in open loop, is accelerated and a bit more damped.

The previous results are not modified by the phygoid, i. e., with a 23rd-order model. Besides, for all models over the operating range, the worst-case stability degree for the phygoid is +0.007 , which is very satisfactory since widely inferior to $0.1 \mathrm{rad} / \mathrm{s}$ that is the limit imposed by specifications.

To illustrate these results, time domain responses of the closed loop between $\mathrm{Nz}_{\text {com }}$ and $\mathrm{Nz}_{\mathrm{CG}}$ are given by Figs. $2 a$ and $2 b$. Let notice that the results without phygoid are rather close to final specifications expected with a feedforward. Then, it is reasonable to think that it will be possible to satisfy the specifications on all models with a simple multimodel feedforward. The state feedback controller is globally (very) satisfactory.

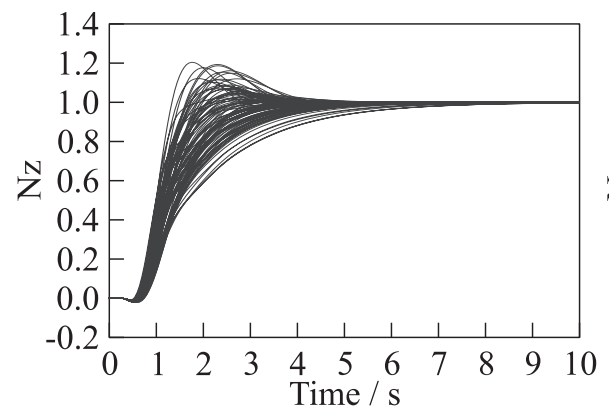

(a)

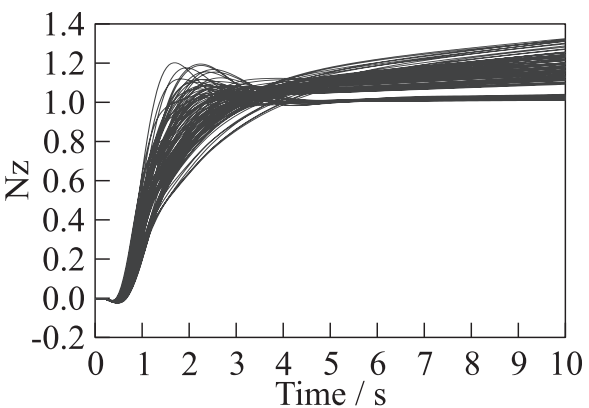

(b)

Figure 2 Time domain response of $\mathrm{Nz}_{\mathrm{CG}}$ for a step input on $\mathrm{Nz}_{\mathrm{com}}$ without $(a)$ and with $(b)$ phygoid

\subsubsection{Design of the observer gain}

The model embedded inside the observed state feedback controller is chosen to be the integral 21-state model (with only a second-order rigid part corresponding to the pitch oscillation mode) + actuator and sensor models. There is no integrator on the $\mathrm{Nz}_{\mathrm{CG}}$ output since this state is directly available for the state feedback controller. Remember that the pitch oscillation mode is correctly damped, so that the observer gain is simply chosen as zero.

The resulting observed state feedback controller is first tested on all models without phygoid mode, for the step response to a filtered wind input. More precisely, a filter $1 /(1+0.05 s)$ is applied to the wind input $w$ and a filter $s /(1+0.05 s)$ is applied to $w^{\prime}$. The result seems satisfactory (Fig. 3). The 
step response to a reference acceleration input is the same as the one obtained with the state feedback controller, and the closed loop poles correspond to those obtained with the state feedback and observer gains, so that they do not need to be checked.

Then, the estimated state feedback controller is applied to all models with phygoid mode:

- as for the closed loop poles, the worst-case stability degree is +0.01951 , which means that the phygoid mode has been essentially stabilized (remember its worst-case open loop value is +0.206 ); and

- the step responses to a reference acceleration input are displayed in Fig. 4.

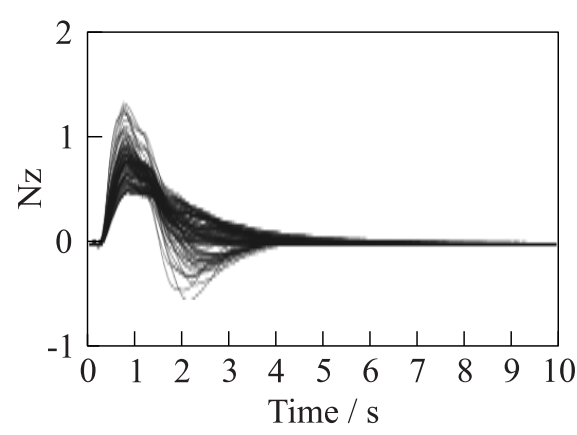

(a)

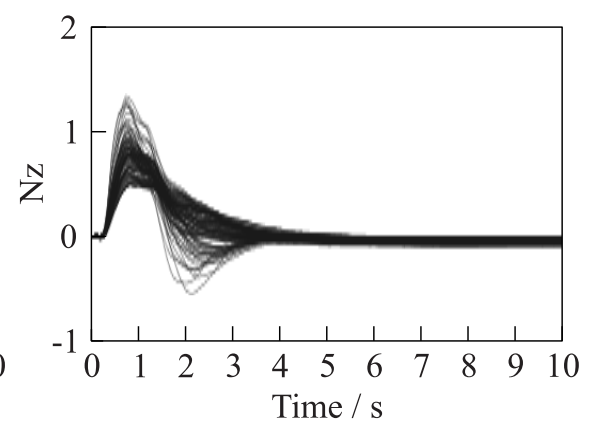

(b)

Figure 3 Step response to the filtered wind input on all models without $(a)$ and with (b) phygoid

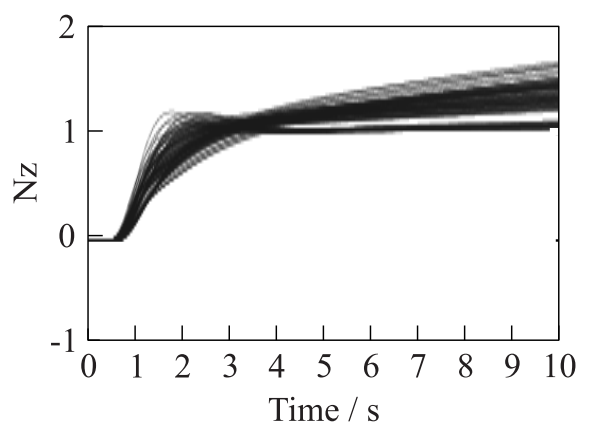

Figure 4 Time domain response of $\mathrm{Nz}_{\mathrm{CG}}$ for a step input on $\mathrm{Nz}_{\text {com }}$ with phygoid mode for all models 


\subsection{Control of the Flexible Part}

Specifications on the flexible part are treated thanks to the Youla parameter design. Let remind that the closed loop transfer functions are parametrized with respect to the Youla parameter of the following way:

$$
T_{w \rightarrow z}=T_{1}-T_{2} Q T_{3}
$$

where $T_{w \rightarrow z}$ represents the closed loop transfer function to minimize or to constraint, $T_{1}$ the initial closed loop transfer function, and $T_{2}$ and $T_{3}$ closed loop transfer functions which depend on the initial stabilizing controller.

The specifications on flexible model are the following ones:

- to minimize the WRMX load level for sizing cases with critical perturbations;

- a command effort to minimize the WRMX compatible with saturations and rate limiters;

- a WRFz preserved with minimization of the WRMX load level; and

- improvement of the passengers comfort.

\subsubsection{Load level alleviation}

The first specification is the main specification and the most difficult one. Typically, the perturbation is either a turbulence or a discrete gust. But generally speaking, the discrete gust is the perturbation which leads to the maximum load level for the WRMX. For discrete gusts, the load level is evaluated as an $l_{\infty}$ norm on the output WRMX for a specific discrete gust. For each flight and mass case, 10 different discrete gusts, which correspond to 10 different amplitudes $U_{d s}$ and scales $H$, are applied. Besides, when the WRMX load level is decreased for one discrete gust, one mass, and one flight case, the load level must represent the maximum load level for all other discrete gusts and flight/fuel cases. In other words, a hard point is to guarantee a maximum load level for all cases. Of course, as indicated previously, it must be done while satisfying saturations and rate limiters with a limited $\mathrm{WRFz}$ load level.

Another and last point is to take into account the $1 \mathrm{~g}$ load. This static load is specific to the longitudinal dynamic and perfectly natural since it corresponds to the compensation of the weight of the aircraft. In brief, the total load level is the result of a static part and a dynamic part. But if the dynamic load is obtained by the linear time domain simulations, it is not the case of the $1 g$ load. For all that, it is the total load which must be minimized and if the same constraint is imposed for all dynamic load, it is not relevant because the total load can be 
very different due to the $1 \mathrm{~g}$ load. A solution is to impose a constraint different for each dynamic load in order to have the same constraint for the total load level.

To decrease the WRMX, load level sizing fuel and flight cases have been determined. Besides, discrete gusts which lead to the highest WRMX load level were determined too. These discrete gusts are called critical discrete gusts. In brief, just sizing flight and fuel cases with critical discrete gusts are used in the optimization problem. But the analysis a posteriori is done with all fuel and flight cases and all discrete gusts.

For Figs. 5-12, the constraints are represented by dotted lines, static load levels by dashed lines, and dynamic or total load levels by solid lines. For an upward discrete gust, the bending moment is negative, that is why the sizing value is represented by the negative part.
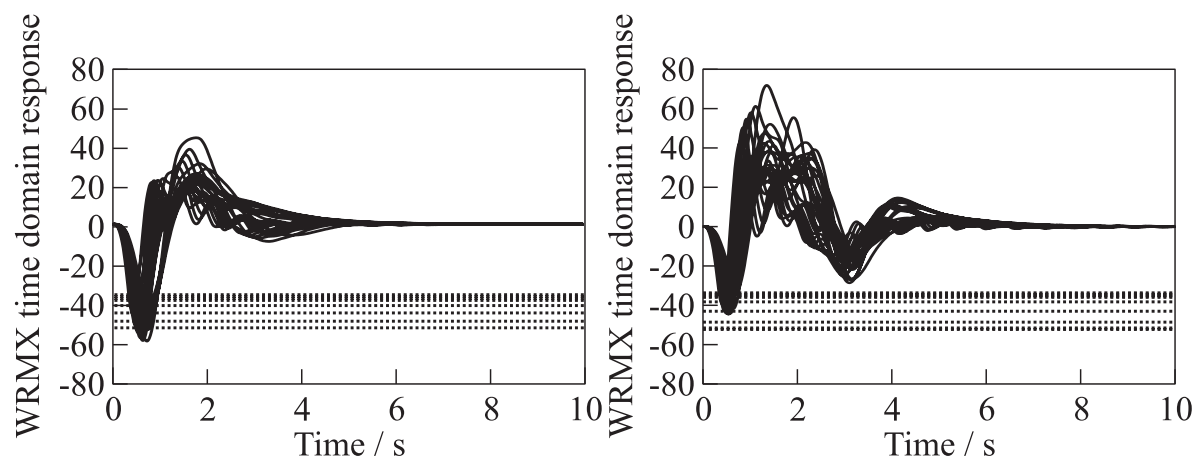

(a)
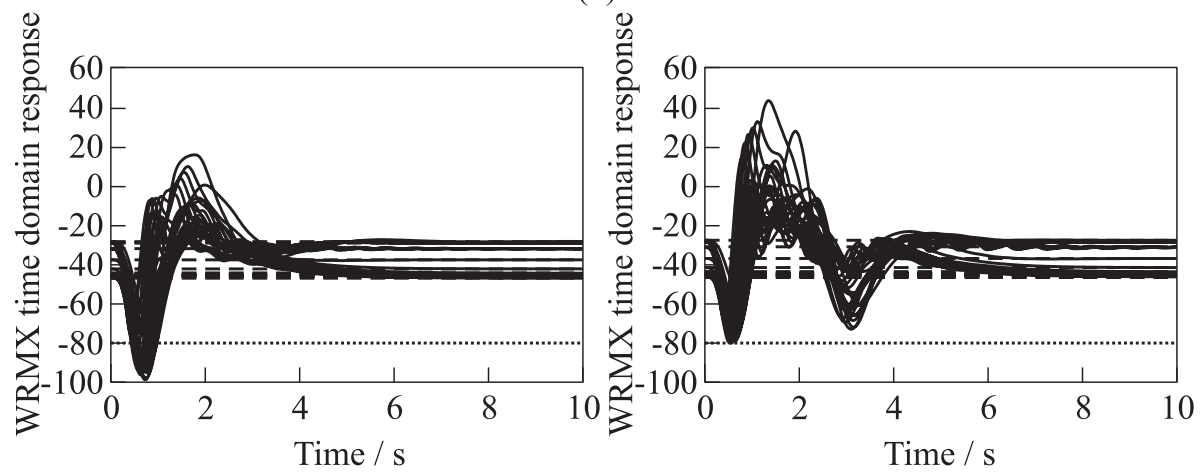

(b)

Figure 5 Time domain response of the WRMX dynamic $(a)$ and total $(b)$ load levels with discrete gust: left column - domain response without Youla parameter; and right column - WRMX time domain response with Youla parameter 


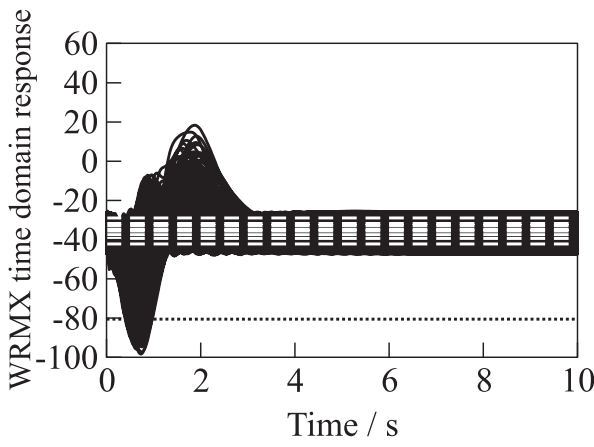

(a)

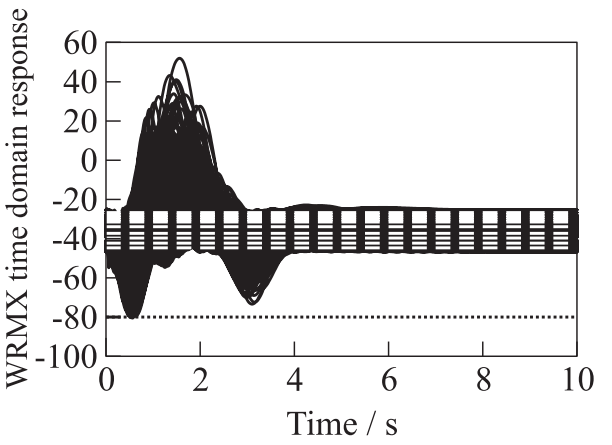

(b)

Figure 6 Time domain response of the WRMX total load level with all discrete gusts and all fuel and mass cases: (a) WRMX time domain response without Youla parameter; and $(b)$ WRMX time domain response with Youla parameter
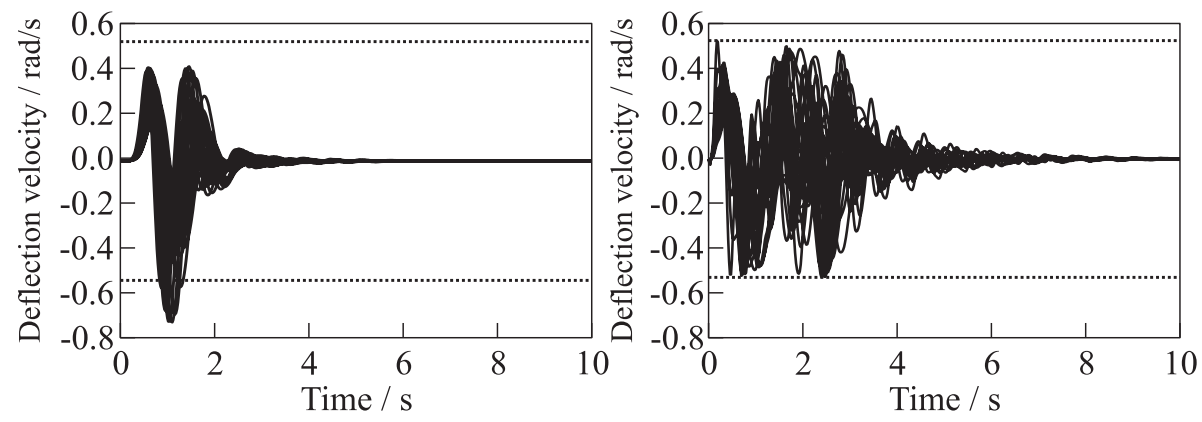

(a)
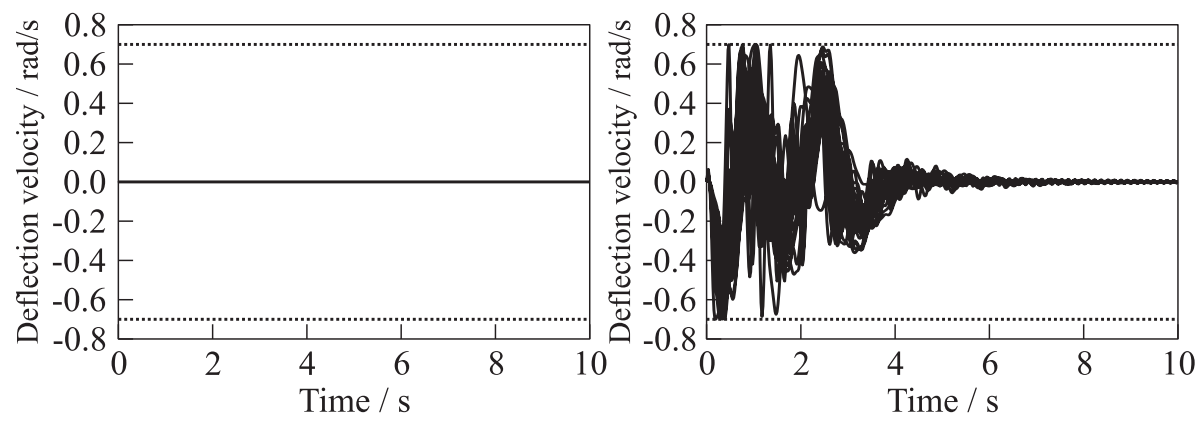

(b)

Figure 7 Deflection velocity of elevators $(a)$ and outer ailerons $(b)$ for sizing mass and flight cases and critical discrete gusts: left column — without Youla parameter; and right column — with Youla parameter 

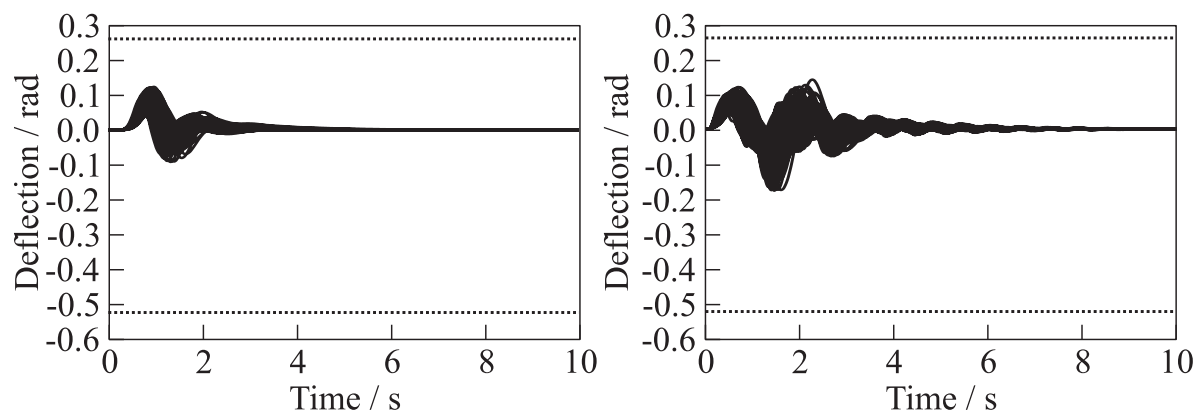

(a)
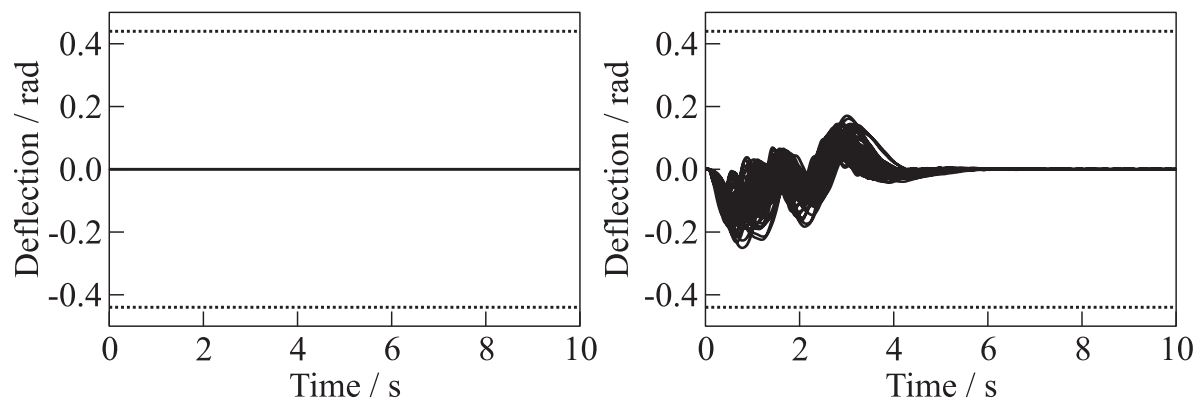

(b)

Figure 8 Deflection of elevators $(a)$ and outer ailerons $(b)$ for sizing mass and flight cases and critical discrete gusts: left column — without Youla parameter; and right column — with Youla parameter

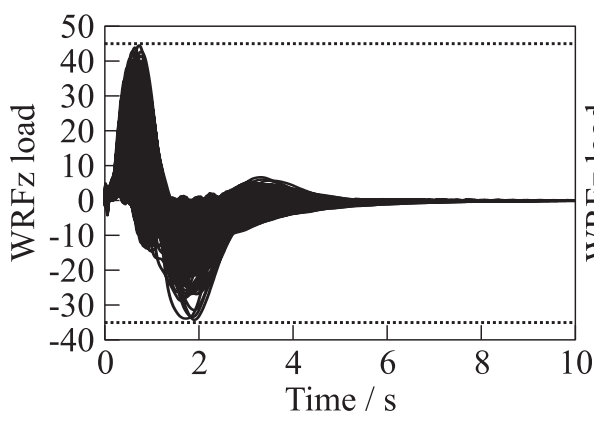

(a)

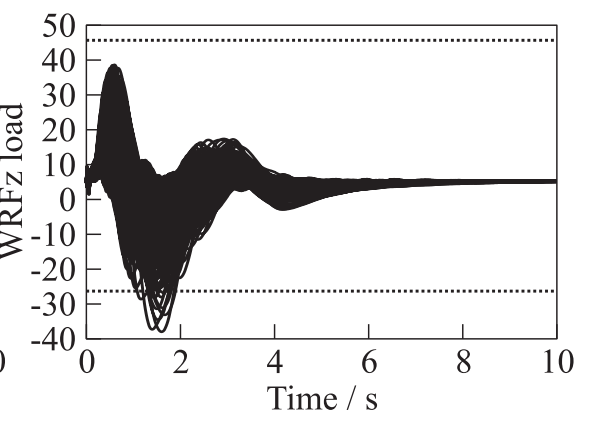

(b)

Figure 9 Time domain response of the WRFz load level with all discrete gusts and all fuel and mass cases: $(a)$ without Youla parameter; and $(b)$ with Youla parameter 
A constraint on the dynamic load is evaluated for each fuel and flight sizing case (Fig. $5 a$, left frame). The Youla pameter is designed and, finally, the result on the dynamic load level is given by Fig. $5 a$ (right frame). The results on total load level are given by Fig. $5 b$ (right frame), from where it is seen that the contraint is the same for all cases (see Fig. $5 b$ ) since the contraint on the dynamic part has been evaluated for this. Finally, a load alleviation of $17 \%$ is obtained on the total load level (see Fig. $5 b$, right frame).

An important point is to check that WRMX load level for all flight and mass cases and all discrete gusts that satisfy the constraints, which represent 81 models $\times 10$ discrete gusts, i. e., 810 time domain simulations for each figure. It is what was represented with Figs. $6 a$ and $6 b$. It is seen from these figures that the constraints are satisfied for all cases.

\subsubsection{Command effort}

Let remind that in the nonlinear scheme, saturations and rate limiters are situated before the actuators and, consequently, on the controller outputs. Then, the signals, which are considered for the synthesis and the analysis, are the controller outputs. Critical constraints are imposed by rate limiters since the deflection velocity before actuator is very high due to limited actuators bandwidth.

In Figs. 7 and 8, deflections and deflection velocities of elevators and outer ailerons are represented for sizing flight and mass cases and critical gusts with respect to time. One may notice that the constraints represented by dotted lines are satisfied. These constraints are given in Table 1 . Let notice that the initial stabilizing controllers whose objective is to satisfy handling qualities does not use ailerons, that is why the result without Youla parameter is 0 .

\subsubsection{Wing root vertical force load level}

A specification concerns the $\mathrm{WRFz}$ which must be preserved with minimization of the WRMX load level.

In Fig. 9, WRFz load level has been represented for all discrete gusts, mass, and flight cases. The dotted lines represent the maximal positive and negative values without Youla parameter. One may notice that the results with Youla parameter are satisfactory since not only the $\mathrm{WRFz}$ is preserved but also, it is decreased for the positive value. The absolute value of the negative part increases but it is not a problem since the $1 \mathrm{~g}$ force is positive.

\subsubsection{Passenger comfort}

Figures $10 a$ and $10 b$ represent comfort cabin with two kinds of filters: $(i)$ seasickness; and $(i i)$ vibration filters. 

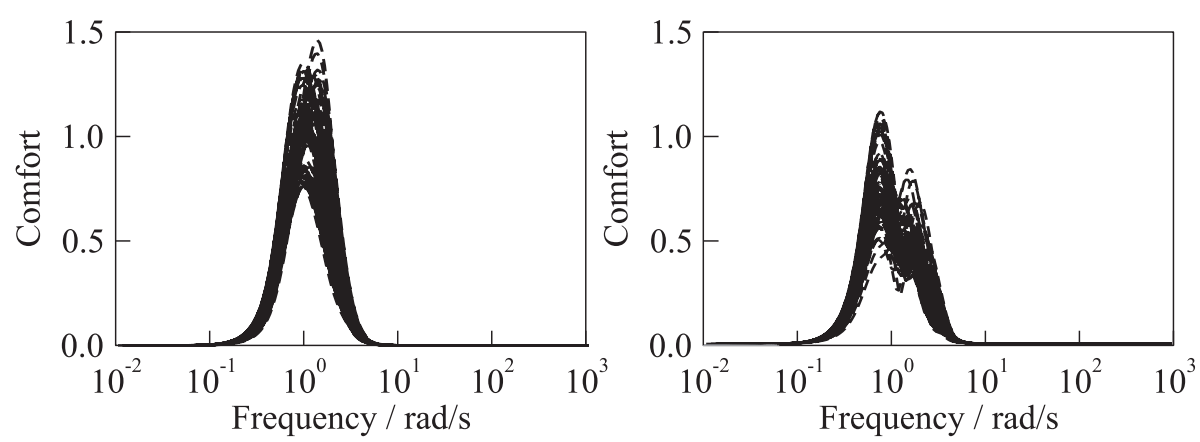

(a)
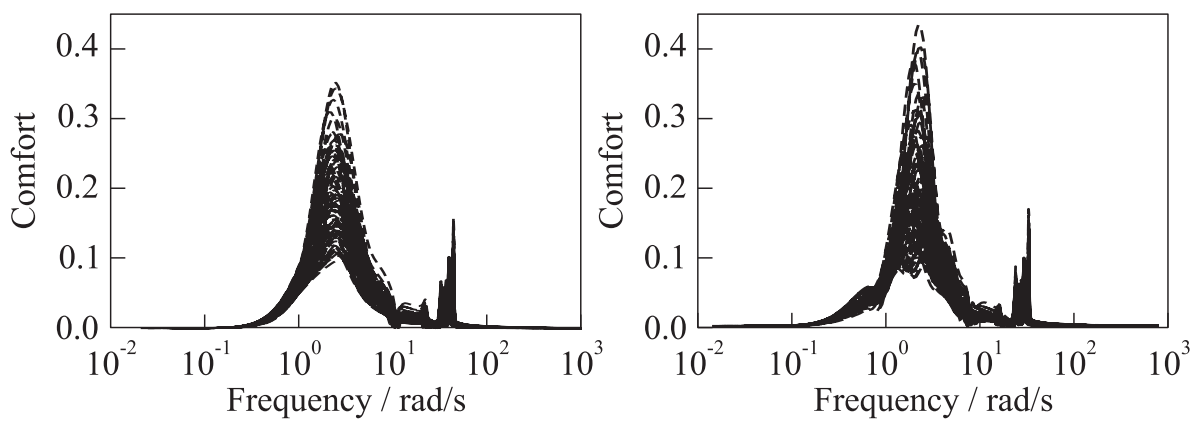

(b)

Figure 10 Transfer functions of comfort cabin with seasickness $(a)$ and vibration $(b)$ filters: left column — without Youla parameter; and right column — with Youla parameter

The comfort criterion is based on the $H_{2}$ norm of the transfer function. Only result with one comfort cabin output has been represented but 5 comfort cabin outputs have been used in the design scheme. In each figure, 81 fuel and flight cases have been represented. The input signal is a white noise filtered by a linearized von Karman filter. Globally, since 5 comfort cabin outputs are used, the $\mathrm{H}_{2}$ norm of $5 \cdot 81=405$ transfer functions are considered.

Certainly, it is not possible to represent all these transfer functions but the global reduction of the $\mathrm{H}_{2}$ norm is of $20 \%$, i. e., the comfort has been improved of $20 \%$. This global reduction can lead to a rise in some transfer functions as it is possible to see in Fig. 10b, right frame.

\subsection{Feedforward}

Let remind handling qualities specifications that have be to satisfied with the feedforward: 


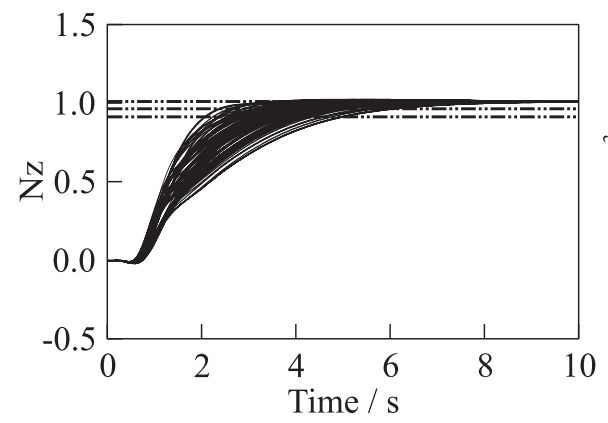

(a)

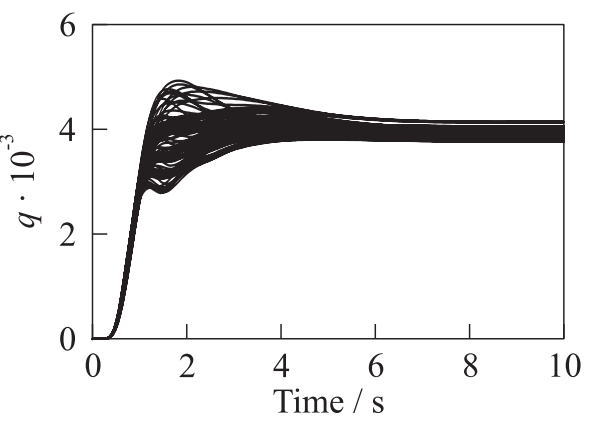

(b)

Figure 11 Step response for $\mathrm{Nz}$ and $q$
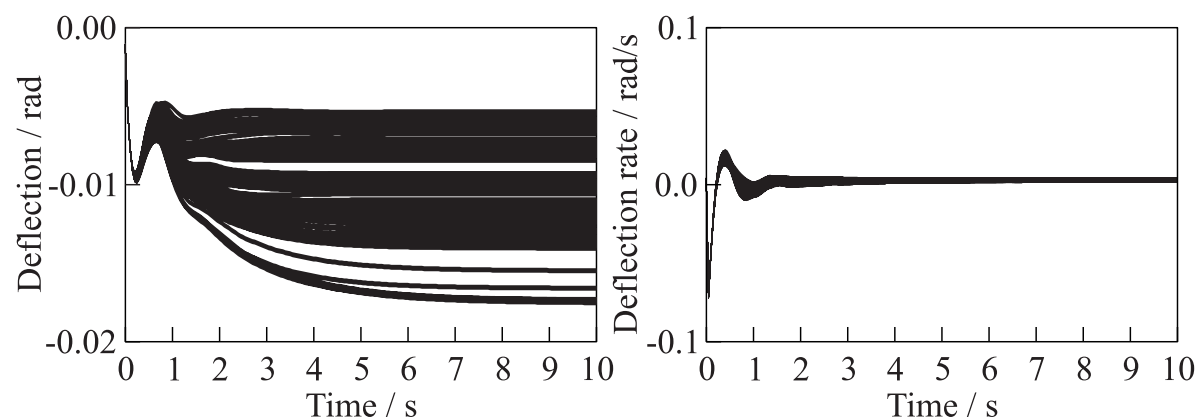

(a)
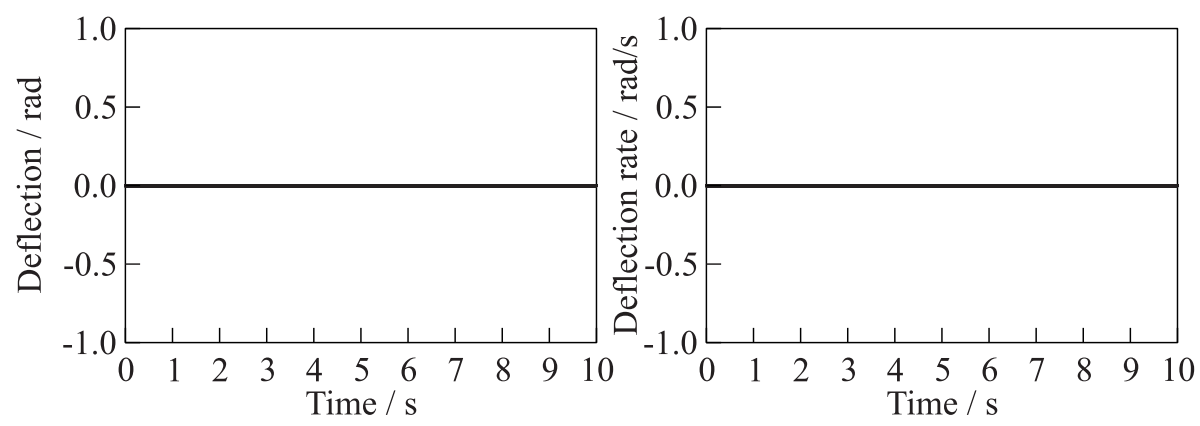

(b)

Figure 12 Step response for $\mathrm{Nz}$ and $q$ : (a) elevators; and (b) ailerons 
- a rising time of 3 to $6 \mathrm{~s}$ is expected with a very limited overshoot on Nz; and

- a maximum overshoot of $30 \%$ on $q$.

Figure 11 represents handling qualities when the feedforward is designed and implemented. One can notice that specifications are fully satisfied now since:

- the overshoot on $\mathrm{Nz}_{\mathrm{CG}}$ is limited to $1 \%$ for the worst case with a mean of $0.45 \%$;

- the rising time on $\mathrm{Nz}_{\mathrm{CG}}$ is $5.95 \mathrm{~s}$ at $95 \%$ of the wanted value or $4.95 \mathrm{~s}$ at $90 \%$ of the wanted value for the worst case. Mean values are of 4.0 and $3.4 \mathrm{~s}$, respestively; and

- the overshoot on $q$ is limited to $21.5 \%$ for the worst case with a mean value of $5.7 \%$.

Besides, this feedforward is multimodel, i.e., a simple tranfer function of order 4 allows to satify specifications for all fuel and flight cases. Of course, all these results on $\mathrm{Nz}_{\mathrm{CG}}$ and $q$ are obtained with a limited command effort since in Fig. 12, it is seen that, first, only elevators are used by the feedforward as shown by Fig. 1 and, second, that deflection and deflection velocity are widely inferior to constraints represented by saturations and rate limiters.

\section{CONCLUDING REMARKS}

In this paper, the results on a BWB aircraft in the context of the European project ACFA have been presented. The methodology to obtain an LPV controller thanks Youla parameter design has been presented. The results on handling qualities are very satisfactory. A simple feedforward is able to shape the time domain response on $\mathrm{Nz}_{\mathrm{CG}}$ to satisfy handling qualities specifications.

The results on flexible part, which consist in minimizing the WRMX load level with discrete gusts, in terms of feasibility have been obtained. This load alleviation has been done while satisfying saturations and rate limiters. Beside, WRFz load level and comfort have been improved.

\section{REFERENCES}

1. Demourant, F., and G. Ferreres. 2002. A frequency domain identification-control approach for a flexible aircraft. IEEE CCA 02.

2. Ferreres, G., and G. Puyou. 2006. Flight control law design for a flexible aircraft: Limits of performance. J. Guidance Control Dyn. 29(4):870-78. 
3. Torralba, J., G. Puyou, and F. Demourant. 2009. Self-scheduling multiobjective control law design for a flexible aircraft. AIAA GNC Conference Proceedings.

4. Boyd, S., and C. Barrat. 1990. Linear controller design: Limits of performance. Prentice Hall.

5. Torralba, J., F. Demourant, G. Puyou, and G. Ferreres. 2009. A method for flexible aircraft LFT modelling. European Control Conference Proceedings.

6. Alazard, D., and P. Apkarian. 1999. Observer-based structures of arbitrary compensators. Int. J. Robust Nonlinear Control 9(2):101-18.

7. Dardenne, I. 1988. Développement de méthodologies pour la synthèse de lois de commande d'un avion de transport souple. Ph.D. Thesis. France: ENSAE. 